\title{
Ximena Erazo, Jaime Esponda y Miguel Yaksic SJ (Eds.). Migración y derechos humanos: mediación social intercultural en el ámbito local. Santiago: Editorial LOM/Fundación Henry Dunant América Latina, 2016, 261 págs.
}

Esta publicación integra 15 artículos que recogen los contenidos de distintas exposiciones realizadas en el Curso de Mediación social intercultural en Asuntos Migratorios, organizado por la Fundación Henry Dunant América Latina junto al Ministerio de Desarrollo Social, y desarrollado entre agosto y octubre de 2015. Los artículos pueden agruparse en cinco grandes temáticas: normativa internacional y nacional, caracterización y subjetividad en torno al fenómeno migratorio, consideraciones en torno a la infancia y mujeres migrantes, participación y ciudadanía y acciones en el ámbito local.

En los primeros tres capítulos del libro, Jaime Esponda, Jorge Martínez y Jaime Gajardo entregan una mirada principalmente desde el Derecho, analizando las distintas normativas sobre migraciones a nivel internacional y nacional, así como las contribuciones de las personas migrantes y el papel que juega la Corte Interamericana de Protección de los Derechos Humanos en Latinoamérica, en materia de migración y derechos humanos.

En primer lugar, Esponda describe una serie de documentos internacionales basados en la Declaración
Universal de los Derechos Humanos, que buscan la protección de los migrantes y que restringen o limitan la autoridad de los Estados en materia de migración. Luego plantea que, en general, los derechos humanos contenidos en los documentos internacionales están recogidos en la Constitución chilena y que ha habido avances en materia de migraciones a nivel nacional. Sin embargo, la legislación migratoria vigente Decreto de Ley 1904 (Ley de Extranjería)- tiene un enfoque restrictivo que regula, principalmente, el régimen de ingreso y permanencia en el país, siendo la más atrasada de América Latina en términos de política pública migratoria y garantía de derechos humanos para migrantes.

En segundo lugar, Martínez expone las diversas contribuciones que implica la migración, entre ellas, el rol en los mercados laborales y el enriquecimiento cultural en una era de globalización y, en contraste, manifiesta que la imposición de barreras a la migración fomenta la irregularidad migratoria y el aumento en la vulnerabilidad de las personas migrantes. En este sentido, es fundamental tener una perspectiva integrada a nivel internacional, especialmente en América Latina. Finalmente, Gajardo menciona algunos casos

\footnotetext{
Ximena Erazo, Jaime Esponda y Miguel Yaksic SJ (Eds.). Migración y derechos humanos: mediación social intercultural en el ámbito local. Santiago: Editorial LOM/Fundación Henry Dunant América Latina, 2016, 261 págs. Tania Toro Spuler

Autoctonía. Revista de Ciencias Sociales e Historia, Vol. II, N¹, Enero-Junio 2018, 159-162. ISSN 0719-8213

DOI: http://dx.doi.org/10.23854/autoc.v2i1.62
} 
que fueron presentados a la Corte Interamericana de Protección de los Derechos Humanos (CIDH) y opiniones consultivas que el organismo ha dado sobre migrantes, a partir de las cuales el autor analiza la doctrina de la CIDH y las obligaciones que ha establecido para los Estados en esta materia.

Por su parte, Eduardo Thayer y Francisco Rivera se centran en las características de la población migrante y la construcción de subjetividades en torno al fenómeno de la migración. Thayer destaca las características de la población migrante en Chile, a partir de los datos de la situación habitacional y de afiliación al sistema de salud de la población migrante (especialmente de las personas que vienen de países de la región). El autor afirma que ambos derechos sociales están siendo vulnerados. Así también, un gran porcentaje de migrantes no cuenta con contrato de trabajo. Esto contrasta con el nivel educacional o años de estudio promedio, que en el caso de la población migrante es entre dos y cinco años más alto que la población chilena, y con la tasa de actividad de la población migrante, que alcanza el 75\%. En este sentido, si se considera que la actividad laboral y la escolaridad son elementos vinculados al desarrollo económico, el aporte que hacen los migrantes al país es muy significativo. Por otro lado, el autor plantea que esta contribución no puede ser el fundamento de reconocimiento y legitimización de la población migrante, pues implica estar sujeto a las condiciones del contexto o la situación particular de cada persona, a riesgo de ponerlos en la figura del "chivo expiatorio" en caso de crisis económica y degradación de los servicios públicos, por ejemplo.

Respectodelacaracterización subjetivadelosmigrantes, por un lado se entiende desde una condición social y, por otro, desde una condición individual. La condición social de migrante está cargada negativamente, pues se asocia a la precariedad de las condiciones de vida, a la discriminación social y, en general, a una situación de exclusión social. Mientras que la condición personal de migrante se asocia al desarraigo, al sacrificio, a la capacidad de adaptación y a la búsqueda de estabilidad y de seguridad. Además, existe la idea, tanto de nativos como de migrantes, de que éstos están en deuda con la sociedad que les ha dado oportunidades de acceso.

Por otro lado, Rivera explora las subjetividades negativas y positivas de vecinos nativos sobre la manifestación del fenómeno migratorio en el territorio que habitan, a partir de una investigación realizada en cinco comunas de la Región Metropolitana. En general, existen buenas relaciones entre los vecinos, quienes se muestran llanos a la convivencia intercultural. No obstante, también están presentes en el imaginario de los nacionales algunos elementos negativos, relacionados con la generalización de estereotipos y prejuicios sustentados en elementos culturales como las costumbres y las condiciones de vida- y fenotípicos de la población migrante. De esta forma, pueden reconocerse dos valoraciones de inmigrantes: el inmigrante 'virtuoso' y el 'vicioso', según los códigos simbólicos de la población nativa asociados a la presencia o ausencia de la temperancia (consumo de drogas lícitas e ilícitas), la trascendencia (religiosidad y vinculación) y la honestidad (disposición al trabajo y al esfuerzo).

Andrea Riedemann, Verónica Vargas y Pamela Walker centran sus artículos en aquellos grupos que proponen como particularmente vulnerables: las mujeres y los niños, niñas y jóvenes migrantes. En primer lugar, Riedemann reflexiona en torno a la necesidad de avanzar hacia una educación intercultural, basándose principalmente en la propuesta de Xavier Besalú (s.f.), quien propone seis aspectos necesarios en los sistemas educativos para ser considerados interculturales y de los cuales el sistema educativo chileno está muy lejos. 
Sin embargo, caben destacar distintas iniciativas hacia este objetivo, como la formación de la Mesa por una Educación Intercultural (EduGlobal, 2017). Verónica Vargas, por su parte, reflexiona sobre la necesidad de incorporar la perspectiva de género y la consideración de la infancia en las intervenciones sociales locales, pues son grupos que están expuestos a situaciones de riesgo distintas a las que sufren los hombres en la tura de movilidad. Esto, dadas las pautas tradicionales y culturales de la sociedad. Para profundizar en la necesidad de la inclusión del enfoque de género en la materia de migración, Walker indaga en las dinámicas familiares y las estrategias de cuidado de niños/as en las familias transnacionales. Este concepto surge de la extensión de lazos entre miembros de un grupo familiar, que deben desarrollar sus relaciones entre su lugar de destino y de origen.

Por su parte, Jaime Esponda y Caterine Galaz reflexionan en torno a las posibilidades de participación y el ejercicio de la ciudadanía de las personas migrantes, entendiendo que lo político constituye un ámbito importante a la hora de mirar el fenómeno de la migración. En primer lugar, Esponda plantea que el ejercicio de la ciudadanía plena para los migrantes -entendida como la "participación efectiva en las decisiones que se adoptan, en los diversos niveles de ejercicio del poder político" (p. 102)- en función de la residencia y no de la nacionalidad, es una garantía para el disfrute de sus derechos humanos, en iguales condiciones que los nativos. Esta idea se sustenta en los principios del Derecho Internacional de los Derechos Humanos y del Derecho Migratorio Internacional, así como en los principios constitucionales de cada Estado. Así también, plantea que esta participación ciudadana plena comienza en los espacios locales, en particular en las comunas, pues son espacios de identificación del individuo con la ciudad. Allí se deben crear espacios de discusión y toma de decisiones que afecten a las comunidades. Esto último es profundizado por Galaz donde además plantea que el reconocimiento es un elemento central de la inclusión social. En este sentido, es necesario actualizar o formular intervenciones y políticas sociales que respondan oportunamente a los derechos, demandas y deberes de las personas migrantes, pero desde la participación social y apertura comunitaria, favoreciendo la promoción del diálogo especialmente vecinal- y el aprendizaje colectivo. Para esto, es preciso que el espíritu de las intervenciones sociales sea sensibilizador, crítico, regulador del etnocentrismo y generadores de nuevos discursos de alteridad, es decir, que cuestionen las nociones instauradas de nación, comunidad, cultura, identidad, racismo, entre otras, y sus manifestaciones cotidianas. En este sentido, la inclusión es un proceso mutuo, no sólo dirigido a las personas migrantes, sino a todos quienes comparten un territorio.

Finalmente, autores como Aguilar, Alzueta, Olave y Dalmazzo rescatan el valor de las acciones locales y destacan la importancia de que sean éstos los ejecutores de las distintas intervenciones y políticas públicas para la inclusión de las personas migrantes.

Pilar Aguilar muestra una revisión del concepto de "buena práctica" y de distintos ejemplos que promueven el ejercicio de los derechos humanos de los migrantes, enfatizando en el rol que juega el municipio en este proceso. Igor Alzueta propone dos ejes de intervención en el ámbito local para generar nuevas relaciones de convivencia y alcanzar la interculturalidad: la implementación de políticas sociales universales y el reconocimiento de la diversidad cultural. Rosa María Olave plantea que los problemas estructurales en el continente de América Latina han generado una dinámica social compleja, donde los conflictos deben considerarse desde una perspectiva multifactorial. Para enfrentar esta situación, la autora propone el uso 
de la mediación social, en particular en los espacios sociales comunitarios, por su potencia emancipadora, transformativa y crítica.

Por su parte, Sylvia Duarte analiza la relación entre el diálogo intercultural y la diversidad, centrándose en el ámbito psicológico y en los procesos de negociación que implica el contacto intercultural. Así también, se enfoca en los procesos de aculturación psicológica y en el papel de la mediación intercultural, tanto para facilitar la inserción de un migrante en una nueva cultura, como para formar ciudadanos capaces de abordar la diversidad humana. Finalmente, Igor Alzueta y Antonia Dalmazzo sistematizan el taller de Mediación Social.

El proceso de aculturación que viven las personas migrantes en Chile, se produce porque deben adaptarse a los patrones y costumbres del país incluso prescindiendo de su marco cultural-, y se sostiene desde el asimilacionismo como paradigma de gestión de la diversidad. Esta perspectiva asume al país de llegada como una sociedad con una cultura hegemónica y homogénea, y está presente en las intervenciones sociales, tanto públicas como privadas[1]. Sin embargo, la diversidad cultural, racial y social que surge a partir de la creciente migración, demanda nuevas perspectivas para el análisis social[2] - Asimismo, requiere cambiar la actual legislación por una con enfoque de derechos, que reconozca y garantice el ejercicio de derechos individuales, sociales, económicos y culturales estipulados en todos los instrumentos internacionales a los que Chile ha adscrito; y que destaquen el rol de la comunidad local, fomentando la vinculación y el sentido de pertenencia -tanto de migrantes como de nativos-, facilitando espacios de encuentro y socialización y reconociendo a las personas como sujetos políticos.

En este libro, aunque no se logra establecer un orden temático cohesionado y coherente, se ofrece un panorama amplio de distintas temáticas que rodean al fenómeno migratorio y se destaca la relevancia de un cambio en la legislación y cultura chilena.

\section{NOTAS}

[1] Francisco Torres, "De la asimilación al pluralismo. Inmigración y gestión de la diversidad cultural en las sociedades contemporáneas". Revista Arxius de Ciències Socials, $\mathrm{n}^{\circ} 11$ (2005): 61-87.

[2] Carolina Stefoni, "Representaciones Culturales y Estereotipos de la Migración Peruana en Chile. Informe final del concurso: Culturas e identidades en América Latina y el Caribe". Programa Regional de Becas CLACSO, 2001. Disponible en la World Wide Web: http://bibliotecavirtual.clacso.org.ar/ar/libros/becas/2000/ stefoni.pdf
Tania Toro Spuler Centro de Estudios Políticos Culturales y Sociales de América Latina -EPOCAL Universidad Bernardo O'Higgins, Chile 